\title{
Further development of synthetic aperture real-time 3D scanning with a rotating phased array
}

Nikolov, Svetoslav; Tomov, Borislav Gueorguiev; Gran, Fredrik; Dufait, R.; Jensen, Jørgen Arendt

Published in:

2003 IEEE Symposium on Ultrasonics Proceedings

Link to article, DOI:

10.1109/ULTSYM.2003.1293286

Publication date:

2003

Document Version

Publisher's PDF, also known as Version of record

Link back to DTU Orbit

Citation (APA):

Nikolov, S., Tomov, B. G., Gran, F., Dufait, R., \& Jensen, J. A. (2003). Further development of synthetic aperture real-time 3D scanning with a rotating phased array. In 2003 IEEE Symposium on Ultrasonics Proceedings (Vol. 2, pp. 1899-1902). IEEE. I E E E International Ultrasonics Symposium. Proceedings https://doi.org/10.1109/ULTSYM.2003.1293286

\section{General rights}

Copyright and moral rights for the publications made accessible in the public portal are retained by the authors and/or other copyright owners and it is a condition of accessing publications that users recognise and abide by the legal requirements associated with these rights.

- Users may download and print one copy of any publication from the public portal for the purpose of private study or research.

- You may not further distribute the material or use it for any profit-making activity or commercial gain

- You may freely distribute the URL identifying the publication in the public portal 


\title{
Further DeVElopment of Synthetic Aperture ReAL-Time 3D SCANNING With A Rotating PHASED ARRAY
}

\author{
Svetoslav Ivanov Nikolov, Borislav Guerguiev Tomov, Fredrik Gran and Jørgen Arendt Jensen \\ Center for Fast Ultrasound Imaging, Bldg 348, Ørsted•DTU, Technical University of Denmark \\ DK-2800 Kgs. Lyngby, Denmark
}

\begin{abstract}
In a previous paper we have presented an approach combining synthetic transmit aperture imaging with a rotating phased array. The method is implemented on a specially made Vermon transducer capable of rotating at $5 \mathrm{~Hz}$. The center frequency of the transducer is $3.2 \mathrm{MHz}$, and the pitch is $0.22 \mathrm{~mm}$. The array active diameter is $16 \mathrm{~mm}$ and the number of elements is 64 . The method is capable of scanning 10 volumes/sec. The order in which the transmit elements were fired made it possible to achieve lateral resolution of a $0.94 \mathrm{~mm}$ at $45 \mathrm{~mm}$ depth, which is comparable to a standard B-mode scan.

The penetration depth was $>10 \mathrm{~cm}$ due to the use of $20 \mu \mathrm{s}$ long frequency modulated (FM) pulses. The dynamic range was limited to $45 \mathrm{~dB}$ because of the non-optimized FM pulse and grating lobes.

This paper presents the work on improving the image quality of the approach: (1) instead of defocusing conventional focusing with an f-number of 1 is used in transmit to create spherical waves, (2) virtual receive elements are synthesized to decrease noise and grating lobes, (3) the compression filter for the FM pulses was modified to suppress the range lobes (4) additional hardware for synchronization is built.
\end{abstract}

\section{OVERVIEW}

Real-time 3-D ultrasound imaging has been the goal of many researchers for over a decade. The main question is how to avoid the tradeoff between speed of acquisition and image quality. Lockwood et al. suggested the use of synthetic transmit aperture ultrasound imaging combined with mechanical motion of a phased array to scan 3-D volumes [1]. In that method, the array is "rocking", and the planes are scanned and beamformed independently one from another using only a few emissions. The use of such a sparse aperture results in high side-lobes.

In 2002, we presented a new approach for acquiring the volume [2]. The transducer is not rocking, but instead it is rotating. The advantage of this method is that more pulseecho events can be used for the beamformation of a single point, thus yielding lower side- and grating-lobes. Figure 1

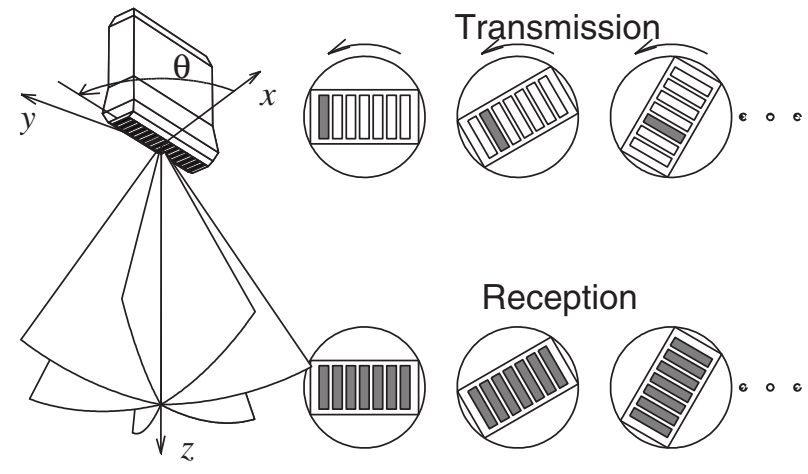

Figure 1: Acquisition and beamformation.

illustrates the acquisition method. A transducer is continuously rotating at a constant angular velocity. A cylindrical wave (such as the wave transmitted by a single element) is transmitted by a group of elements. This is symbolized on the figure with a dark filling color. All elements are used in receive. A point in the volume is formed by a simple delayand-sum beamformation:

$$
\mathbf{H}(\vec{x})=\sum_{i \in \Omega_{x m t}(\vec{x})} \sum_{j=1}^{N} a_{i j}(\vec{x}) r_{i j}\left(t\left(\vec{x}, \vec{x}_{i}, \vec{x}_{j}\right)\right),
$$

where $\vec{x}$ are the coordinates of the point being formed, $\Omega_{x m t}(\vec{x})$ is the set of transmissions, in which the cylindrical wave has passed through the imaged point, $\vec{x}_{i}$ is the center of the transmitted spherical wave, $\vec{x}_{j}$ is the position of the receiving element $j, r_{i j}(t)$ is the signal received by element $j$ at transmission $i$, and $N$ is the number of elements in the transducer. The time instance $t\left(\vec{x}, \vec{x}_{i}, \vec{x}_{j}\right)$, at which a sample for the sum is taken, corresponds to the propagation time from the center of transmission $\vec{x}_{i}$ to the beamformed point $\vec{x}$ and back to the transducer element $\vec{x}_{j}$ :

$$
t=\frac{1}{c}\left(\left|\vec{x}-\vec{x}_{i}\right|+\left|\vec{x}_{j}-\vec{x}\right|\right),
$$


where $c$ is the speed of sound.

The created images have a spatial resolution in the azimuth plane comparable to that of a standard B-mode scan [2]. The dynamic range, however, was limited to $45 \mathrm{~dB}$, which is less than conventional images. In order for a scan method to be adopted for imaging, it must not only offer new advantages, but must perform in all other aspects at least as good as the existing methods. Several areas were identified as candidates for optimization:

- The generation of spherical waves. When the method was first implemented, the spherical waves were created by defocusing $[1,2,3]$. A similar effect could be achieved with focusing instead $[4,5]$.

- Optimization of the FM chirps. The pulses and filters used in [2] were not designed using the impulse response of the system as suggested in [6].

- The synchronization. The scans relied on the simultaneous start of the scan and the rotation of the transducer. Additional hardware for synchronization was to be built

- Reduce the grating-lobe level by creating virtual receive elements, whose directivity pattern has a narrow main lobe.

These topics are considered in the same order in the following sections of the paper.

\section{SPHERICAL WAVE GENERATION}

Creating spherical waves is essential for the proposed method. There are two ways of generating spherical waves using defocused or focused transmission. A defocused transmission with 13 transmit elements and a $20 \mu$ s chirp was used in [2]. This resulted in a penetration depth of up to $120 \mathrm{~mm}$. Using a focused transmission has the advantage of increasing the energy sent into the body, thus increasing the penetration depth. Fig. 2 (left) shows the propagation path which must be used in the delay calculation for the beamfomration process [4]. The time at which a sample must be taken becomes (2):

$$
t=\frac{1}{c}\left(\left|\vec{x}_{i}-\vec{x}_{o}\right|+\left|\vec{x}-\vec{x}_{i}\right|+\left|\vec{x}_{j}-\vec{x}\right|\right),
$$

where the focal point is considered to be the virtual element $\vec{x}_{i}$, and $\vec{x}_{o}$ is the center of the aperture.

The result of using focused transmits is shown in the Fig. 2 (right). The spherical wave was created using 15 transmit elements. The focusing is at a depth of $5 \mathrm{~mm}$ in front of the transducer. A conventional 2 cycles sine pulse is used as an excitation in transmit. The cysts are detectable up to a depth of $140 \mathrm{~mm}$, which is an increase of more than $20 \mathrm{~mm}$ compared to [2].

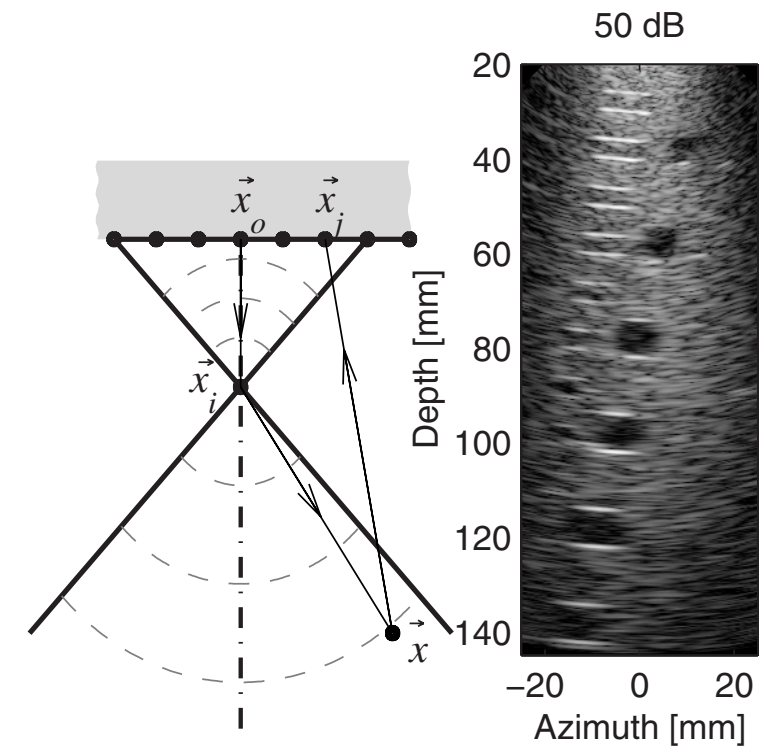

Figure 2: Delay calculations geometry (left). A B-mode slice created with a conventional pulse (right).

\section{OPTIMIZATION OF CHIRPS}

In order to attain a better SNR codes are deployed. Coded excitation has previously been investigated in [6]. A linear FM chirp has been used as excitation,

$$
s(t)=a(t) \sin \left(2 \pi f_{0} t+\frac{B}{2 T} t^{2}\right),-\frac{T}{2} \leq t \leq \frac{T}{2},
$$

where $a(t)$ is a temporal weighting function, $f_{0}$ is the center frequency, $B$ is the bandwidth being swept and $T$ is the duration of the chirp. To achieve an acceptable axial resolution, a compression filter has to be used. Normally a matched filter with a temporal weighting function is applied

$$
f(t)=g(t) s(-t) .
$$

However, the signal which is received at the transducer is in fact given by

$$
r(t)=s(t) * h_{t i}(t) * h_{t r}(t),
$$

where $h_{t i}(t)$ is the pulse echo impulse response of the interrogated tissue and $h_{t r}(t)$ is the pulse echo impulse response of the transducer. If the transducer pulse echo impulse response is known, one can compensate for it and replace (5) with,

$$
\tilde{f}(t)=g(t) \tilde{s}(-t),
$$

where $\tilde{s}(t)=s(t) * h_{t r}(t)$. The side lobes are then reduced below $-50 \mathrm{~dB}$ as shown in Fig. 3. 


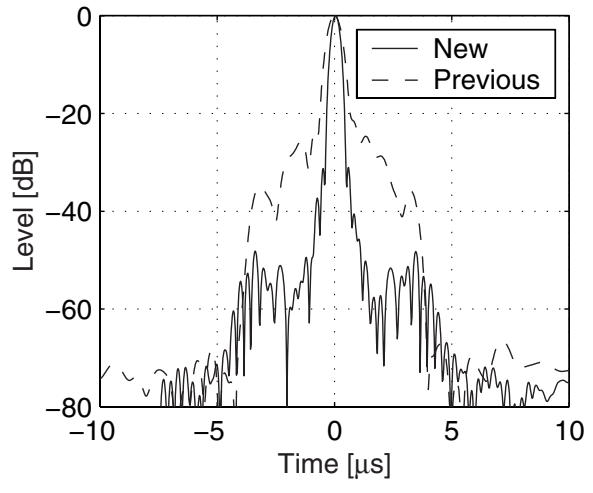

Figure 3: Reduction of the range side lobes with the new filter design.

\section{SYNCHRONIZATION}

The Vermon (Cedex, France) phased array rotating transducer is driven by a DC motor and controller supplied by Maxon Motor AG (Sachseln, Germany). The position of the rotating block is indicated by a rotation encoder and an end switch. The two sensors in the encoding block generate differential signals. An illustration of the positive signals from both sensors is shown in Fig. 4 (top part). The direction of rotation determines which signal precedes the other.

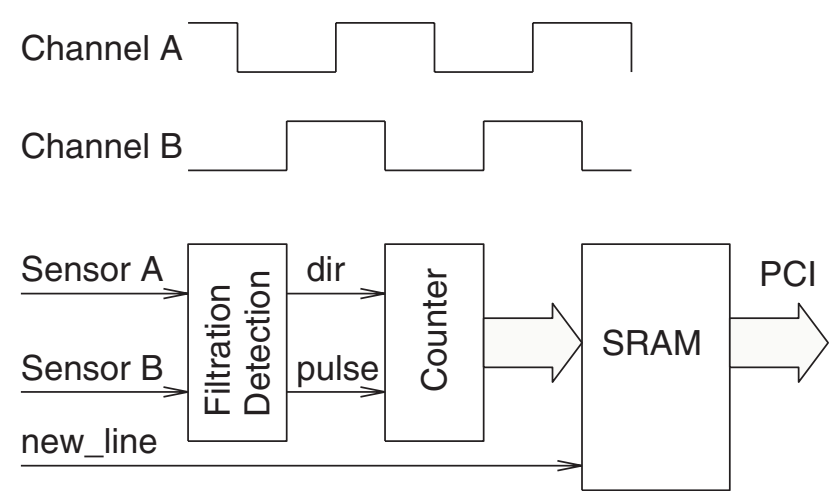

Figure 4: Rotary encoder sensor signals

An angle detector was built using the XC2S_EVAL board form CESYS GmbH (Herzogenaurach, Germany). The board features a PCI interface, Xilinx Spartan-2 FPGA and a SRAM block. The structure of the detector is shown in Fig. 4 (bottom part).

In that setup, the new_line signal is provided by a detection logic connected to one of the transmitter channels. The positive signals from both sensors are monitored and their relation is used to determine the position and the increment of a counter. Whenever a new image line acquisition is initiated, the present value of the counter is stored in the SRAM. After the end of the acquisition, the stored values are read through the PCI interface in a Linux environment. The error in the recorded position is less than $\pm 0.3^{\circ}$.

\section{VIRTUAL RECEIVE ELEMENTS}

For the different points in the image, a different number of transmissions is used as shown in Eq. 1. The points, which are close to the axis of rotation will be formed using a larger number of transmit events, and the points lying at the outskirts will be created with a smaller number of transmissions. The transmission order can be arranged such that the resolution of the image at all points is the same [4]. The side- and grating-lobe levels are, however, higher, when the number of emissions is less.

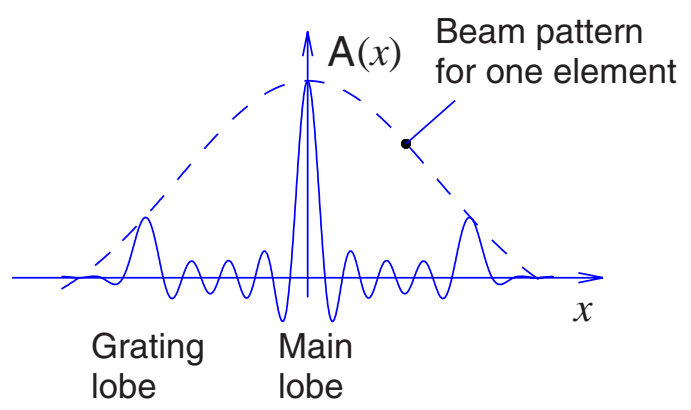

Figure 5: Illustration of suppression of the grating lobes

Fig. 5 shows how the grating lobes are suppressed. The distance between the grating lobes and the main lobe is inversely proportional to the number of elements in the aperture. The level of the grating lobes is suppressed by the radiation pattern of a single element. The bigger the element is, the lower the grating lobe level is.

One way to decrease the grating lobes is to synthesize receive elements, which are large and have a narrow directivity pattern. The distance between the centers of the receive elements must be maintained at $\lambda / 2$. The synthesis of receive elements is done by summing the signals from a number of elements prior to the beamformation. In order to maintain the sensitivity for the different directions in the image, the signals are delayed such that the synthesized element "looks" along scan line which will be beamformed (very much like a sunflower). Fig. 6 illustrates how a virtual receive element is synthesized. The dark dots mark the positions of the physical elements. The pale lines are the synthesized receive elements. The delays of the active elements, which are used to create one of the virtual receive elements are adjusted so as to align on the lines symbolizing the receive elements. If the currently beamformed line is perpendicular to the transducer 


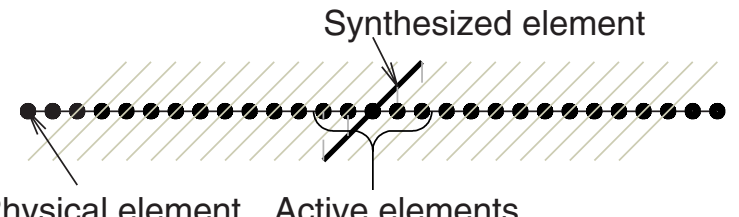

Physical element Active elements

Figure 6: Illustration of the virtual receive elements
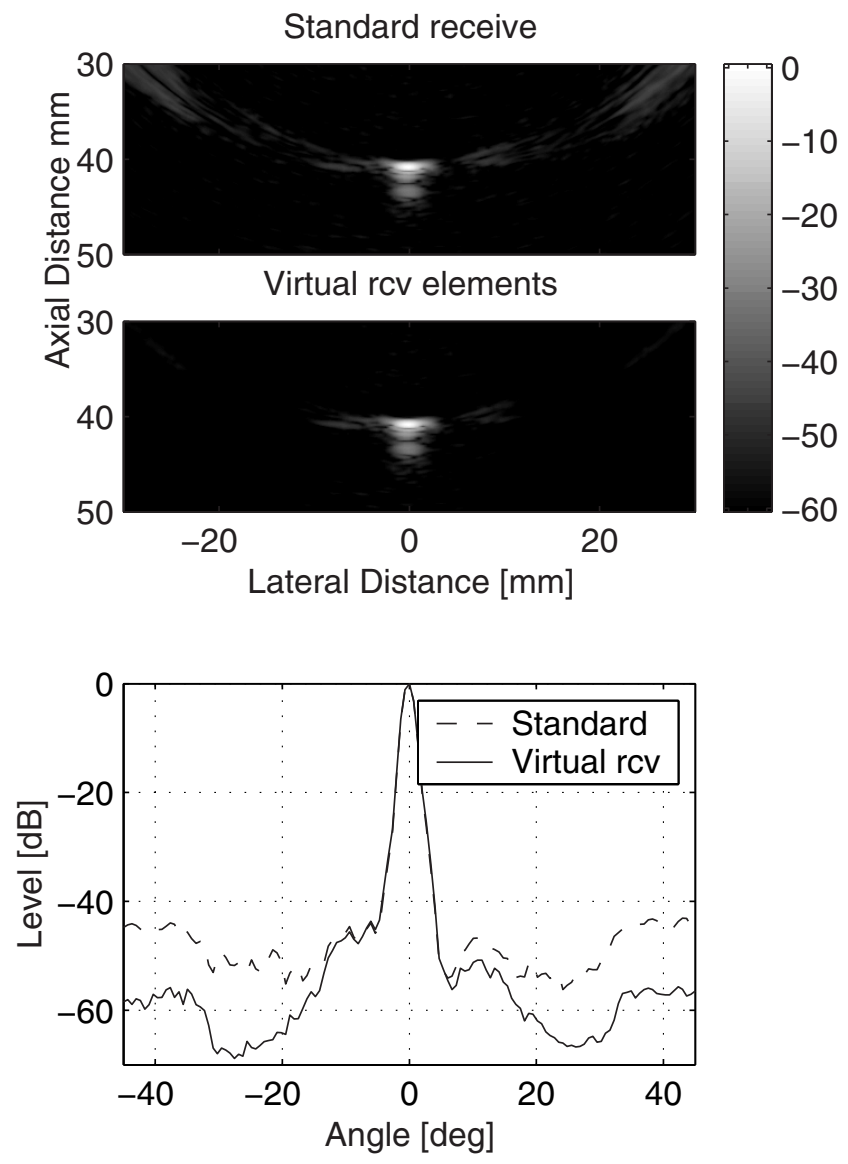

Figure 7: Sidelobe reduction using virtual receive elements.

surface, then synthesizing the receive elements is simply summing the received signals. If the beamformed line is tilted at an angle, then the signals must be appropriately delayed.

Figure 7 shows the reduction in the side lobe level when virtual receive elements are used. The image is created using only 13 emissions, which is the number of emissions normally used to create the points at the outskirts of the volume. Five elements were used in receive to generate a virtual receive element. The plot shows that the side- and grating-lobe level is reduced with more than $10 \mathrm{~dB}$, which will correspondingly increase the contrast of the acquired images.

\section{CONCLUSION}

The 3D synthetic aperture imaging technique with a rotating array is capable of scanning 10 volumes per second. Previously it was shown that the images had resolution as the conventional B-mode images.

This paper presented the work on increasing the dynamic range and penetration depth of the images. The use of focused transmits allows to scan up to $140 \mathrm{~mm}$ with conventional sinusoidal pulses. The use of chirps increases the scan depth. The careful filter design suppresses the range side lobes to below $-50 \mathrm{~dB}$. The use of virtual receive elements reduces the grating lobes for the points at the edge of the volume with more than $10 \mathrm{~dB}$. The method, thus, has the ability of generating $3 \mathrm{D}$ scans whose quality matches the quality of the conventional 2D scans.

\section{ACKNOWLEDGMENTS}

This work was supported by grant 9700883, 9700563 and 26-01-0178 from the Danish Science Foundation and by BK Medical A/S Denmark.

\section{REFERENCES}

[1] G. R. Lockwood, J. R. Talman, and S. S. Brunke. Realtime 3-D ultrasound imaging using sparse synthetic aperture beamforming. IEEE Trans. Ultrason., Ferroelec., Freq. Contr., 45:980-988, 1998.

[2] S. I. Nikolov, R. Dufait, A. Schoisswohl, and J. A. Jensen. Three-dimensional real-time synthetic aperture imaging using a rotating phased array transducer. In Proc. IEEE Ultrason. Symp., pages 1545-1548, 2002.

[3] M. Karaman, P. C. Li, and M. O'Donnell. Synthetic aperture imaging for small scale systems. IEEE Trans. Ultrason., Ferroelec., Freq. Contr., 42:429-442, 1995.

[4] S. I. Nikolov and J. A. Jensen. Virtual ultrasound sources in high-resolution ultrasound imaging. In Proc. SPIE Progress in biomedical optics and imaging, volume 3, pages 395-405, 2002.

[5] M. H. Bae and M. K. Jeong. A study of synthetic-aperture imaging with virtual source elements in B-mode ultrasound imaging systems. In IEEE Trans. Ultrason., Ferroelec., Freq. Contr., volume 47, pages 1510-1519, 2000.

[6] T. X. Misaridis and J. A. Jensen. An effective coded excitation scheme based on a predistorted FM signal and an optimized digital filter. In Proc. IEEE Ultrason. Symp., volume 2, pages 1589-1593, 1999. 